\title{
Microfluidic Systems for the Belousov-Zhabotinsky Reaction
}

\author{
Brent T. Ginn, Bettina Steinbock, Murat Kahveci, and Oliver Steinbock* \\ Department of Chemistry and Biochemistry, Florida State University, Tallahassee, Florida 32306-4390
}

Received: July 1, 2003; In Final Form: November 26, 2003

\begin{abstract}
We describe an experimental methodology for the study of chemical self-organization in micropatterned reaction systems. Our approach is based on office-printer-assisted soft lithography and allows the fabrication of centimeter-scale devices with reactor units as small as $50 \mu \mathrm{m}$. The devices are made from the elastomeric material poly(dimethylsiloxane) and are filled with a modified Belousov-Zhabotinsky solution. This excitable reaction-diffusion medium employs 1,4-cyclohexanedione as a bubble-free organic substrate and Fe(II)$\left[\text { batho }\left(\mathrm{SO}_{3}\right)_{2}\right]_{3}$ as a high-absorbance redox catalyst/indicator. Chemical wave propagation is affected by the loss of bromine from the aqueous phase into the elastomer matrix. The strength of this activating process depends on the local surface-to-volume ratio and can increase the wave velocity by a factor of 2 . For devices with gridlike reactor networks, we observe a pronounced deformation of target patterns and the pinning of spiral waves to single elastomer obstacles as well as to obstacle clusters.
\end{abstract}

\section{Introduction}

Many physical, chemical, and biological systems self-organize concentration patterns that are characteristic for excitable reaction-diffusion media. ${ }^{1,2}$ Important examples include rotating spiral waves and expanding circular wave trains that are commonly referred to as target patterns..$^{2-4}$ These dissipative structures result from local reaction processes that involve the rapid, autocatalytic production of an activator species if the concentration of the activator exceeds a certain threshold. The spatial coupling is mediated by diffusion that induces an activator flux into the vicinity of a superthreshold perturbation. In response, a concentration pulse forms that propagates through the system at constant speed and constant amplitude. These excitation waves annihilate upon collision because of increased threshold values in the wake of the pulse and, hence, differ significantly from most waves in conservative systems.

Important examples of excitable reaction-diffusion (RD) media include the $\mathrm{CO}$ oxidation on platinum $^{5}$ and the $\mathrm{Be}$ lousov-Zhabotinsky (BZ) reaction. ${ }^{6}$ The latter reaction involves the oxidation of an organic substrate by bromate in acidic solution and employs redox catalysts such as $\mathrm{Ce}(\mathrm{III}) / \mathrm{Ce}(\mathrm{IV})$ and $\mathrm{Fe}(\mathrm{II})(\text { phen })_{3} / \mathrm{Fe}(\mathrm{III})(\text { phen })_{3}$. Most chemical RD systems are spatially homogeneous in the sense that their kinetic parameters are uniform. Excitation waves in living organisms, however, are affected by the heterogeneous character of the medium. For instance, calcium waves in single oocytes are influenced by the microscopic architecture of the cell. ${ }^{7}$ Furthermore, concentration waves in cell tissue and cell colonies, such as aggregating slime mold amoebae, show profound dependencies on heterogeneous cell distribution. ${ }^{8,9}$ It is therefore not surprising that recent research on pattern formation in chemical reaction systems has focused on the implementation of heterogeneous media, and it is expected that these efforts will yield important models for the study of various complex phenomena in biology. Moreover, it has been suggested that nonlinear reaction systems with tailored heterogeneities can be used for data storage and processing as well as for the creation of highly parallel computing devices. ${ }^{10-12}$

* To whom correspondence should be addressed. E-mail: steinbck@ chem.fsu.edu. Phone: 850-644-4824. Fax: 850-644-8281.
Most of the experimental effort toward the fabrication of RD systems with controlled heterogeneities has focused on the $\mathrm{CO}$ oxidation on platinum crystals and the BZ reaction. For example, Wolff et al. developed techniques to modify the surface catalytic activity of $\mathrm{Pt}(110)$ single-crystal surfaces in real time and space with an addressable laser beam. ${ }^{13}$ For the same system, photolithography was used to create microscopic, reacting domains on platinum surfaces with titanium and palladium overlayers. ${ }^{14,15}$ Moreover, several methodologies have been developed for the BZ reaction. These include the use of glass capillaries connecting large reaction compartments, ${ }^{16}$ microemulsions, ${ }^{17}$ the inkjet-controlled deposition of the catalyst onto membranes, ${ }^{18}$ and the projection of light patterns onto the photosensitive $\left[\mathrm{Ru}(\mathrm{bpy})_{3}\right]^{2+}$-catalyzed reaction. ${ }^{19-21}$ Suzuki et al. presented perhaps the most promising approach to the creation of static microscale-patterned BZ systems. ${ }^{22-24}$ They utilized photolithography to control the diffusion of the BZ catalyst ferroin into gel systems that eventually served as the $\mathrm{BZ}$ reaction matrices.

Although lithography-assisted techniques are ideally suited to the creation of micropatterned BZ reactors, Suzuki's approach suffers from diffusion broadening of the catalyst patterns. Here, we describe an alternative methodology that utilizes soft lithography for the fabrication of poly(dimethylsiloxane) reactors. It follows in part techniques developed by Whitesides et al. ${ }^{25,26}$ that are frequently employed in microfluidics. However, the specific features of micrometer-scale BZ experiments in closed reaction systems constitute particular problems that relate to the detrimental formation of gaseous products and the difficult detection of low-absorbance concentration patterns. These problems are solved by employing specifically suited substitutes of the classic organic substrate and redox indicator. In the following, we present a detailed description of the diverse aspects of our newly developed methodologies and utilize these techniques for the study of target patterns and spiral waves in gridlike microreactors.

\section{Materials and Equipment}

Photomasks are produced using Kodak kodalith ortho 35$\mathrm{mm}$ black and white film, Kodak fixer, Kodak kodalith super 
RT developer (B\&H Photo), a Nikon FM10 SLR camera with a Nikon Micro-NIKKOR 55-mm lens, and a copy stand equipped with two 500-W photolamps. We utilize 3-in.-diameter $\times 0.5$-mm-thick polished silicon wafers (Silicon Inc.) as a substrate for template production via photolithography. The wafers are etched prior to use with concentrated sulfuric acid (Fisher) and hydrogen peroxide (Acros, 35\%). SU-8 2100 negative radiation sensitive resist, SU-8 developer (Micro Chem Corp.), and 2-propanol (Fisher) are employed in template production. The resist is diluted with cyclopentanone (Acros) prior to use and applied with a Specialty Coating Systems Inc. P-6000 spin coater. An Oriel 1000-W quartz-tungsten-halogen (QTH) lamp serves as the light source during lithography. For silanization of the silicon wafer, (tridecafluoro-1,1,2,2-tetrahydrooctyl)-1-trichlorosilane (United Chemical Technologies Inc.) is used. Silicon elastomer kits (Fisher, Sylgard 184) are purchased for the fabrication of the final microfluidic devices. All profilometric measurements are performed with a KLATencor P-15 profilometer. Devices are cleaned with $100 \%$ ethanol (Florida Distillers Co.) and exposed to air plasma in a Harrick PDC-32G Plasma Cleaner.

Aqueous solutions of 1,4-cyclohexanedione (Aldrich), sodium bromate (Fluka), and bromine (Fisher) are prepared in Nanopure water $(18 \mathrm{M} \Omega \mathrm{cm})$ from a Barnstead EASYpure UV unit. Standardized $5 \mathrm{M}$ sulfuric acid (Sigma-Aldrich) and ferroin solution (Acros, $0.025 \mathrm{M}$ tris(1,10-phenanthroline)iron(II) sulfate) are used without further purification. $\mathrm{Fe}(\mathrm{II})\left[\mathrm{batho}\left(\mathrm{SO}_{3}\right)_{2}\right]_{3}$ is prepared in $25 \mathrm{mM}$ sulfuric acid by dissolving bathophenanthrolinedisulfonic acid (Acros) and ferrous sulfate heptahydrate (Fluka) in a stoichiometric ratio of 3:1 to yield the complex at a concentration of $25 \mathrm{mM}$. UV/vis absorbance spectra are recorded with a diode array spectrophotometer (HewlettPackard, HP8453).

In our BZ experiments, chemical waves are observed optically using a monochromatic charged-coupled-device (CCD) camera (COHU 2122; $640 \times 480$ pixels at 8 bits). Data are acquired on a computer as bitmap images using commercial software (HLImage++97) and a low-noise frame grabber (Data Translation, DT3155). Image analysis is preformed with programs developed in an IDL 5.2 programming environment (Research Systems Inc.).

\section{Fabrication of BZ Microchips}

Masters. The first step in the preparation of the microstructured reaction systems (Figure 1) is the design of the desired pattern using a vector-based graphics program. The pattern is then printed on white paper using a laser jet printer and transferred photographically to a high-contrast black and white film. Next, the film is developed and cut to yield the desired photomasks. Black and white areas on the original print out correspond to transparent and black areas on the photomasks, respectively. The transmittance of unexposed photonegatives varies between 78.3 and $82.6 \%$ in the relevant wavelength range of 350 to $400 \mathrm{~nm}$, whereas the light-exposed areas of the masks fully block UV/vis light.

The templates or "masters" used for the creation of the microfluidic devices are produced by soft lithography. Polished silicon wafers are used as the substrate for these templates. The silicon wafer is etched in a 70/30\% v/v mixture of concentrated sulfuric acid and hydrogen peroxide, respectively, for $5 \mathrm{~min}$. The etched wafer is then removed, rinsed thoroughly with Nanopure water, and dried with compressed air. The wafer is spin-coated with diluted SU-8 2100 resist (1:10 weight ratio of cyclopentanone and resist, respectively) at $500 \mathrm{rpm}$ for $5 \mathrm{~s}$ and

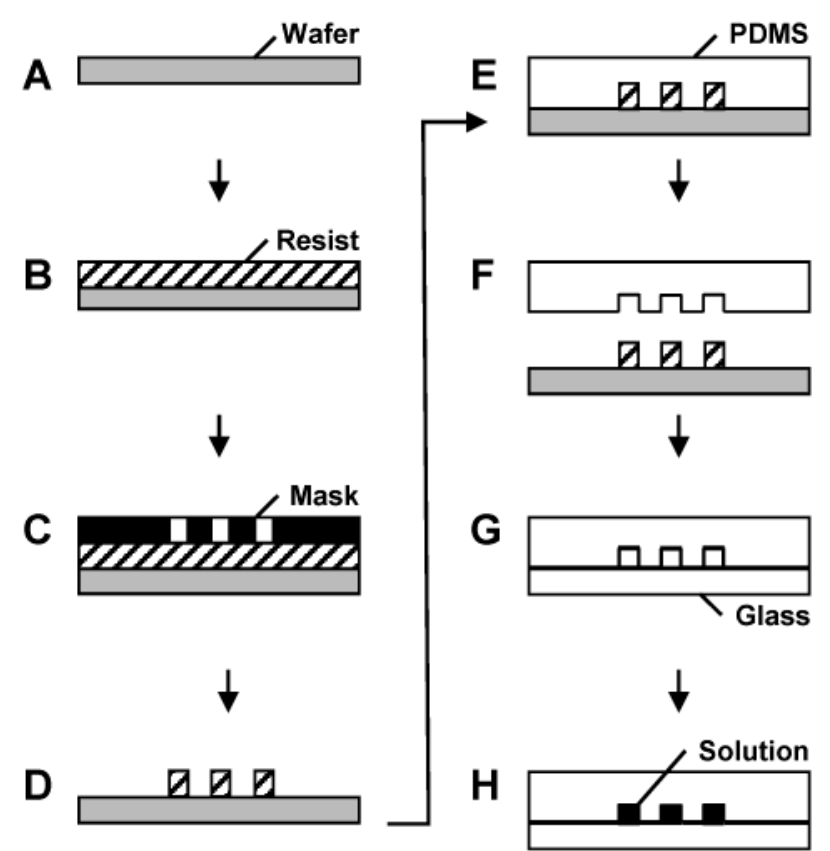

Figure 1. Schematic drawing of master production by soft lithography $(\mathrm{A}-\mathrm{D})$ and device fabrication $(\mathrm{E}-\mathrm{H})$. A single side polished silicon wafer (A) is coated with SU-8 2100 resist and prebaked (B). The photomask is applied to the wafer, exposed to light, and then postbaked (C). The sample is developed and silanized (D). Unpolymerized PDMS is poured over the master (E). After polymerization, the PDMS is peeled from the master $(\mathrm{F})$. The device is cleaned, exposed to air plasma, and sealed to a microscope slide $(\mathrm{G})$. In the last step, the microreactor $(\mathrm{H})$ is filled with $\mathrm{BZ}$ solution.

then at $1000 \mathrm{rpm}$ for $35 \mathrm{~s}$. The coated wafer is then prebaked at $65^{\circ} \mathrm{C}$ on a contact hot plate for $5 \mathrm{~min}$ and then soft baked at $95{ }^{\circ} \mathrm{C}$ for $20 \mathrm{~min}$. Next, the desired photonegative is placed directly in contact with the resist layer, and an aluminum shielding is used to protect the resist not covered by the photonegative. The sample is then exposed to the unfiltered light of a QTH lamp for $1 \mathrm{~min}$ at a distance of $8 \mathrm{in}$. After exposure, the wafer is baked at $65^{\circ} \mathrm{C}$ for $1 \mathrm{~min}$ and then at $95^{\circ} \mathrm{C}$ for 10 min. It is air cooled for 5 minutes and then placed in a bath of SU-8 developer for $10 \mathrm{~min}$. The wafer is rinsed with 2-propanol to remove any developer. The result is a master bearing a surface relief of the original pattern. The height of this relief is approximately $80 \mu \mathrm{m}$. Slight modifications of the preparation allow this height to be adjusted in the range of 50 to $250 \mu \mathrm{m}$.

PDMS Molds. The actual microfluidic devices used in our experiments are produced from the elastomeric material poly(dimethylsiloxane) (PDMS) (Figure 1E-H). The master is first coated with (tridecafluoro-1,1,2,2-tetrahydrooctyl)-1-trichlorosilane to prevent the adhesion of PDMS to the template pattern. The PDMS elastomer is prepared from the Sylgard 184 base and the curing agent (weight ratio 10:1). The mixture is degassed in a vacuum desiccator until all air bubbles have been removed, poured over the master in a Petri dish, and then degassed for an additional $30 \mathrm{~min}$. Next, the Petri dish is placed on a hot plate at $55{ }^{\circ} \mathrm{C}$ for $24 \mathrm{~h}$ to allow polymerization of the PDMS. After cooling the sample to room temperature, the polymer is carefully peeled from the surface of the master. The result is a polymer with the negative surface relief of the master. Excess PDMS surrounding the device is cut away using a straight razor. The device is then cleaned by a sequence of rinses in Nanopure water, ethanol, and $2 \mathrm{M}$ sulfuric acid to remove any unincorporated polymer from the surface.

Before loading the microfluidic device with solution, it must be sealed with an appropriate planar surface. We employ 


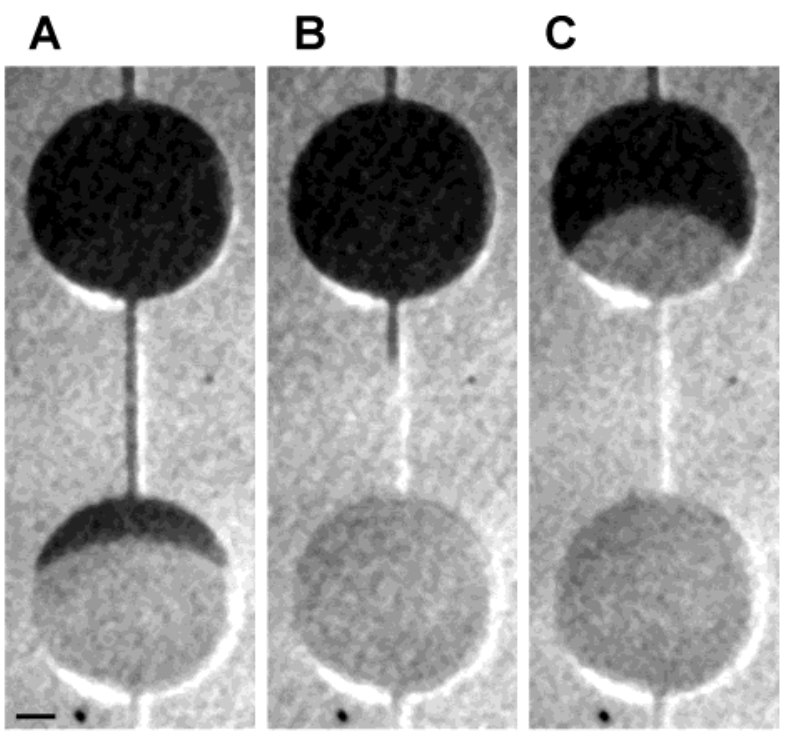

Figure 2. Oxidation wave traveling through a BZ system in a patterned microreactor. The channel has a height of $80 \mu \mathrm{m}$ and a width of 50 $\mu \mathrm{m}$. The circular portion of the reactor has a diameter of $1 \mathrm{~mm}$, and the length of the reference bar (A) is $200 \mu \mathrm{m}$. Initial BZ concentrations are $1.50 \mathrm{M} \mathrm{H}_{2} \mathrm{SO}_{4}, 0.15 \mathrm{M} \mathrm{CHD}, 0.10 \mathrm{M} \mathrm{NaBrO}_{3}$, and $3.1 \mathrm{mM}$ ferroin. The snapshots are separated by $10 \mathrm{~s}$.

two different sealing methods, mechanical and chemical. For simple devices in the fashion of straight channels, we use mechanical sealing. The mechanical seal is accomplished by setting the device between two plexiglass plates that are then secured together using screws. One of the plates bears two holes so that the device may be filled with solution. The solution is manually injected into the device using a disposable syringe and a tube attached to a valve leading into the plexiglass. The chemical seal is accomplished through chemical modification of the PDMS surface. A freshly cleaned device is exposed to air plasma for $45 \mathrm{~s}$ in a plasma cleaner. The sample is then placed on a clean microscope slide for $2 \mathrm{~h}$, allowing the PDMS surface to seal covalently to the glass. The device is then filled with solution by vacuum injection. We find that the chemical seal is the best method for complex devices because of its ability to produce an irreversible liquid-tight seal that allows these reactors to be filled without the accumulation of air bubbles.

Cleaning the microfluidic devices after each use is essential to prevent the build up of chemical residue on the PDMS surface. The cleaning procedure involves rinsing the device with Nanopure water, $2 \mathrm{M}$ sulfuric acid, and ethanol and then drying with compressed air. For devices that are mechanically sealed, this is easily done, whereas for plasma-sealed PDMS devices cleaning is accomplished by repeated vacuum injections.

BZ Waves in Thin Channels. Figure 2 shows three consecutive snapshots of a chemical wave propagating through a microstructured PDMS device. Bright and dark regions correspond to oxidized and reduced states of the ferroin-catalyzed CHD-BZ system, respectively. The excitable area of the device consists of circular domains (diameter $1 \mathrm{~mm}$ ) connected by narrow channels (width $50 \mu \mathrm{m}$ ). In this example, the autocatalytic reaction front travels from the bottom portion of the image area upward. Upon exiting the narrow channels, it assumes a curved shape and traverses the circular domain. Notice that the use of 1,4-cyclohexanedione as the organic substrate prevents the formation of undesired gas bubbles. ${ }^{27-29}$

The use of conventional absorption detection as demonstrated in Figure 2 becomes increasingly difficult as the device is further miniaturized. Moreover, it is preferable to employ low concen-
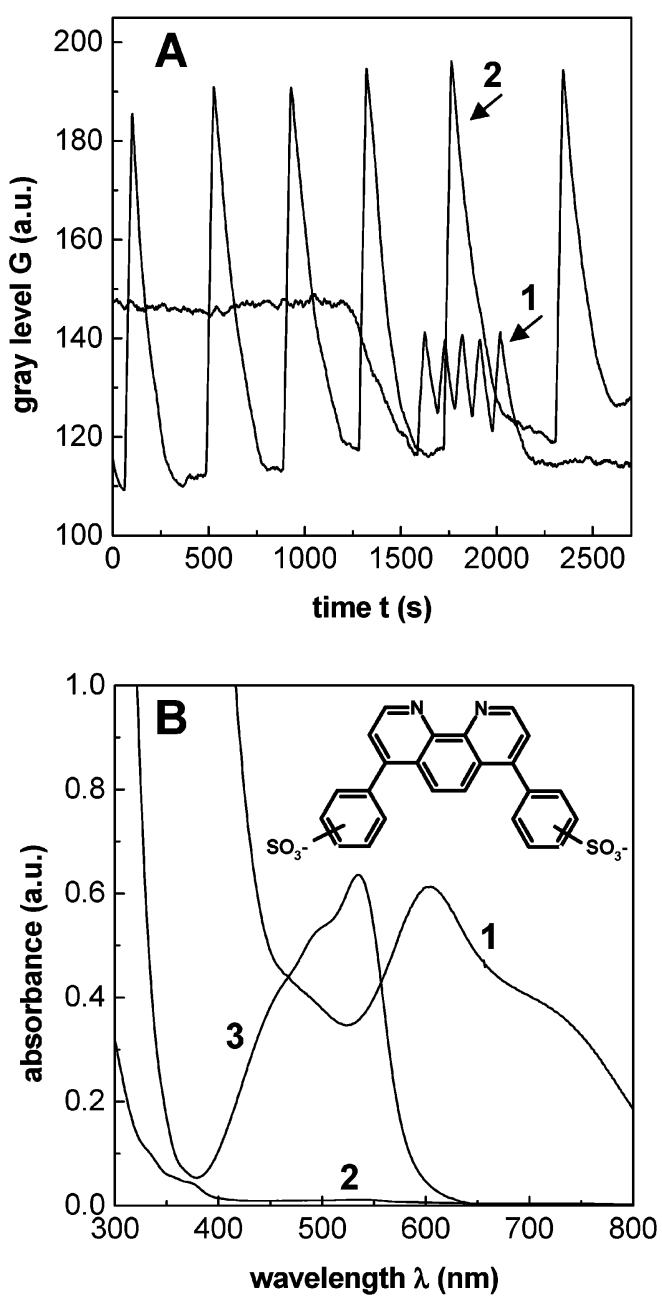

Figure 3. (A) Gray levels $G$ as a function of time $t$ from wave propagation through straight channel microreactors with a height of $80 \mu \mathrm{m}$. The two catalysts that are used are (1) ferroin and (2) Fe(II)[batho $\left.\left(\mathrm{SO}_{3}\right)_{2}\right]_{3}$. Initial $\mathrm{BZ}$ concentrations are $0.50 \mathrm{M} \mathrm{H}_{2} \mathrm{SO}_{4}, 0.15 \mathrm{M}$ CHD, $0.10 \mathrm{M} \mathrm{NaBrO}_{3}$, and $3.1 \mathrm{mM}$ ferroin or $\mathrm{Fe}(\mathrm{II})\left[\mathrm{batho}\left(\mathrm{SO}_{3}\right)_{2}\right]_{3}$. The ferroin system is homogeneously oxdizied during the first $1200 \mathrm{~s}$ of the data shown. (B) Absorbance for oxidized and reduced states of $\mathrm{Fe}$ (II) $\left[\text { batho }\left(\mathrm{SO}_{3}\right)_{2}\right]_{3}$. The catalyst concentration in $(2,3)$ is $33 \mu \mathrm{M}$ and yields low absorbance for the oxidized form. The concentration of the oxidized complex in (1) is significantly higher $(350 \mu \mathrm{M})$. The structure of the ligand batho $\left(\mathrm{SO}_{3}\right)_{2}$ is shown in the upper right corner.

trations of the catalyst because such conditions increase the lifetime of the chemical patterns within the closed reaction system. Because the catalyst also acts as the indicator species, a decrease in its concentration diminishes the absorption signal further. A possible solution to this problem is the use of redox catalysts with a more pronounced difference in the molar absorptivity of the reduced and oxidized forms. In this context, $\mathrm{Fe}(\mathrm{II})\left[\text { batho }\left(\mathrm{SO}_{3}\right)_{2}\right]_{3}$ is a suitable substitute for ferroin and also has a sufficiently high solubility under the acidic conditions presented by the $\mathrm{BZ}$ reaction. ${ }^{30,31}$

Figure 3A shows typical intensity signals of chemical waves in an 80- $\mu \mathrm{m}$-high channel loaded with ferroin-catalyzed and $\mathrm{Fe}$ (II) [batho $\left.\left(\mathrm{SO}_{3}\right)_{2}\right]_{3}$-catalyzed $\mathrm{BZ}$ solutions. With our 8-bit $\mathrm{CCD}$ camera and for white-light illumination, we could achieve fewer than 20 gray levels of separation between the oxidized and reduced states of the ferroin system (curve 1). In the case of $\mathrm{Fe}(\mathrm{II})\left[\text { batho }\left(\mathrm{SO}_{3}\right)_{2}\right]_{3}$, however, our setup yields 70 gray levels of separation between the two states (curve 2). This example demonstrates that the use of $\mathrm{Fe}(\mathrm{II})\left[\mathrm{batho}\left(\mathrm{SO}_{3}\right)_{2}\right]_{3}$ indeed yields the desired enhancement of the absorption signal generated by 
TABLE 1: Catalysts for the Belousov-Zhabotinsky Reaction

\begin{tabular}{|c|c|c|c|c|c|c|}
\hline \multirow[b]{2}{*}{ catalyst } & \multirow[b]{2}{*}{ ligand name } & \multirow[b]{2}{*}{$\begin{array}{l}\text { charge } \\
\text { ox/red }\end{array}$} & \multicolumn{2}{|c|}{ reduced } & \multicolumn{2}{|c|}{ oxidized } \\
\hline & & & $\begin{array}{l}\lambda_{\max } \\
(\mathrm{nm})\end{array}$ & $\begin{array}{c}\epsilon_{\lambda \max } \\
\left(\mathrm{M}^{-1} \mathrm{~cm}^{-1}\right)\end{array}$ & $\begin{array}{l}\lambda_{\max } \\
(\mathrm{nm})\end{array}$ & $\begin{array}{c}\epsilon_{\lambda \max } \\
\left(\mathrm{M}^{-1} \mathrm{~cm}^{-1}\right)\end{array}$ \\
\hline $\mathrm{Fe}(\text { phen })_{3}$ & 1,10-phenanthroline & $+3 /+2$ & 510 & 11000 & 590 & 600 \\
\hline $\mathrm{Fe}\left[\text { batho }\left(\mathrm{SO}_{3}\right)_{2}\right]_{3}$ & $\begin{array}{l}\text { 4,7-diphenyl-1,10- } \\
\text { phenanthroline disulfonate }\end{array}$ & $-3 /-4$ & 535 & 22100 & 602 & 1800 \\
\hline
\end{tabular}

traveling waves. Moreover, we observe that the relaxation in the wake of an excitation pulse is significantly slower in the case of the $\mathrm{Fe}(\mathrm{II})\left[\text { batho }\left(\mathrm{SO}_{3}\right)_{2}\right]_{3}$-catalyzed reaction.

The absorption spectra of $\mathrm{Fe}(\mathrm{II})\left[\mathrm{batho}\left(\mathrm{SO}_{3}\right)_{2}\right]_{3}$ and $\mathrm{Fe}(\mathrm{III})-$ [batho $\left.\left(\mathrm{SO}_{3}\right)_{2}\right]_{3}$ are shown in Figure 3B. The Figure presents three curves that correspond to the oxidized (labeled 1 and 2) and the reduced state (labeled 3 ) of the catalyst. Spectra 2 and 3 are obtained for concentration of $33 \mu \mathrm{M}$, whereas spectrum 1 is measured at a concentration of $350 \mu \mathrm{M}$. The reduced state has a deep-red color with $\lambda_{\max }=535 \mathrm{~nm}$, and the oxidized state appears green with $\lambda_{\max }=602 \mathrm{~nm}$. (See Table 1 for additional data.) The drastic difference in absorbance extends over the entire visible region of the electromagnetic spectrum and makes the two states easily distinguishable. On the basis of these measurements, we suggest that unfiltered white light is an adequate type of illumination for absorption measurements of BZ systems containing this catalyst.

Front Velocities and Bromine Transport. For a given set of reagent concentrations, we observed that the wave speed shows a pronounced dependence on the width of the PDMSbound channels. For example, we found that the front shown in Figure 2 decelerates abruptly as it enters the large, circular domains from the connected narrow channels. This phenomenon persists in the case of the $\mathrm{Fe}(\mathrm{II})\left[\mathrm{batho}\left(\mathrm{SO}_{3}\right)_{2}\right]_{3}$-catalyzed reaction system and for a variety of different geometries of the microfluidic devices.

To quantify this intriguing effect, we measured the wave front velocity in a wedge-shaped channel. An optical micrograph of such a dye-filled channel is shown in Figure 4A. The width of the channel increases continuously from 0.20 to $1.00 \mathrm{~mm}$ over a distance of $20 \mathrm{~mm}$, whereas the height is constant at $80 \mu \mathrm{m}$. Figure 4B shows a time-space plot of chemical waves propagating through this microdevice. The Figure is generated by piling up the consecutive gray-level profiles. Consequently, each oxidation pulse creates one continuous bright band within the time-space plot. The slope of the leading edge of these bands corresponds to the inverse propagation velocity. The waves reveal a pronounced increase in velocity as they traverse narrower segments of the wedge-shaped channel. Notice that this effect weakens as the interpulse distances decrease between subsequent oxidation pulses.

We analyzed the velocity of the first oxidation pulse by extracting its position as a function of time and by calculating the temporal derivative of these data. Figure 5 shows that the front velocity increases steadily from approximately 7 to 14 $\mathrm{mm} / \mathrm{min}$. This pronounced dependence stems from the specific nature of the boundary between the PDMS matrix and the aqueous BZ solution. The results indicate that the excitable medium is experiencing activation in the case of reaction systems with a large surface-to-volume ratio. We suggest that the primary source of this activation is the transport of bromine from the aqueous phase into the PDMS elastomer. This passive removal of bromine induces a decrease in the concentration of bromide, which is known to be the key inhibitor species in the
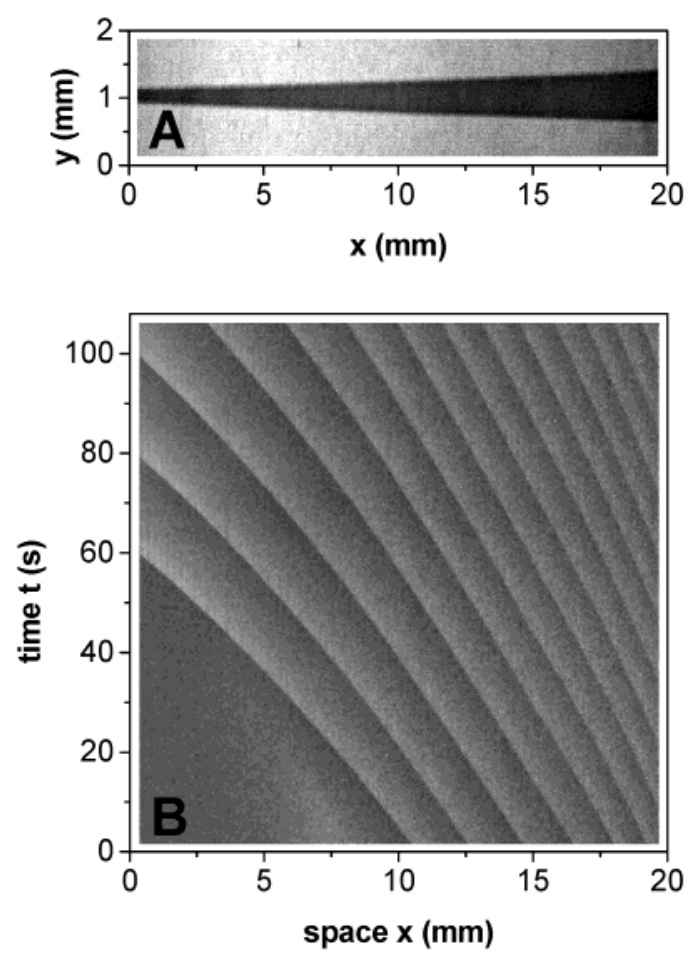

Figure 4. (A) Micrograph of a wedge-shaped microreactor filled with $25 \mathrm{mM}$ ferroin solution. (B) Time-space plot of ferroin-catalyzed BZ waves traversing the wedge-shaped microreactor. The waves accelerate because of changes in the width of the reactor track. The experiment is carried out with an initial $\mathrm{BZ}$ concentration set of $1.50 \mathrm{M} \mathrm{H}_{2} \mathrm{SO}_{4}$, $0.15 \mathrm{M} \mathrm{CHD}, 0.10 \mathrm{M} \mathrm{NaBrO}_{3}$, and $3.1 \mathrm{mM}$ ferroin.

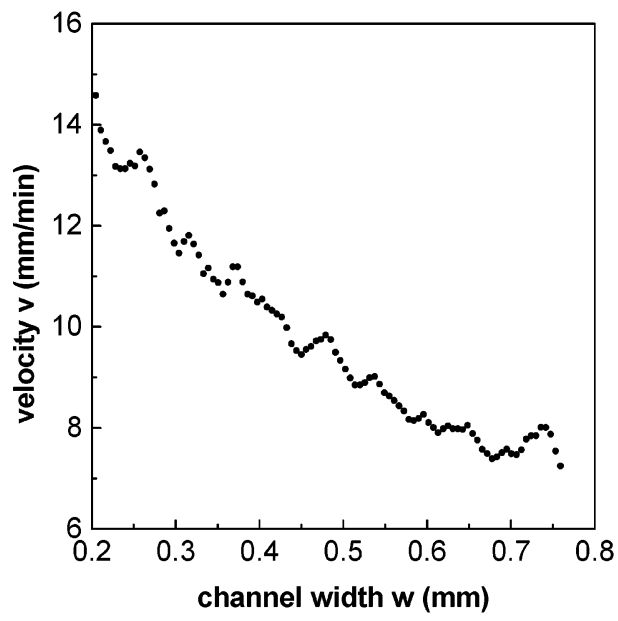

Figure 5. Wave front velocities as a function of channel width for experiments performed in a wedge-shaped microreactor (cf. Figure 4).

$\mathrm{BZ}$ reaction. ${ }^{32}$ The dynamic coupling of bromide and bromine is the result of the fast equilibrium

$$
5 \mathrm{Br}^{-}+\mathrm{BrO}_{3}^{-}+6 \mathrm{H}^{+} \leftrightarrow 3 \mathrm{Br}_{2}+3 \mathrm{H}_{2} \mathrm{O}
$$

To test the hypothesis of bromine loss into the PDMS, we carried out measurements of the relevant partition coefficient. 


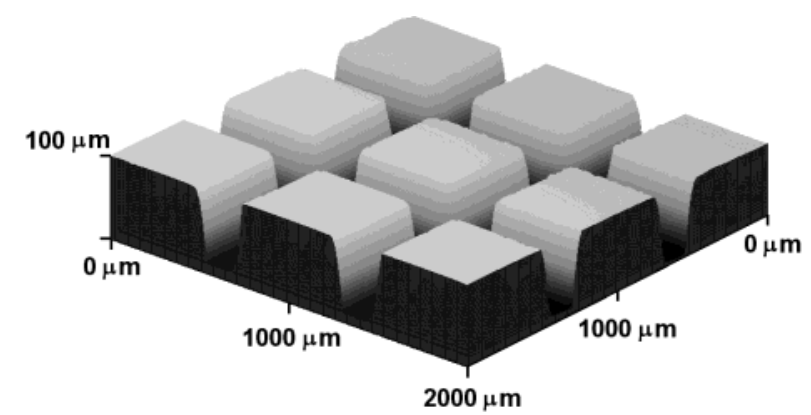

Figure 6. Profilometric scan of a $2 \times 2 \mathrm{~mm}^{2}$ area of a grid-style PDMS device before sealing.

In these experiments, thin PDMS samples were submerged in an aqueous bromine solution. The solution was highly acidic ( $1 \mathrm{M} \mathrm{H}_{2} \mathrm{SO}_{4}$ ) to avoid the formation of other bromine species. After $48 \mathrm{~h}$, the residual bromine concentration in the aqueous phase was measured spectrophotometrically. For a temperature of $25^{\circ} \mathrm{C}$, the data agreed well with the expected dependence $A^{(\mathrm{aq})} /\left(A^{(0)}-A^{(\mathrm{aq})}\right)=(1 / \kappa) V^{(\mathrm{aq})} / V^{(\mathrm{PDMS})}$, where $A^{(0)}, A^{(\mathrm{aq})}, V^{(\mathrm{PDMS})}$, $V^{(\mathrm{aq})}$, and $\kappa$ denote the initial and residual absorbance of the aqueous bromine solution, the volumes of the two sample phases, and the partition coefficient, respectively. These preliminary measurements yield an effective partition coefficient $\kappa=\left[\mathrm{Br}_{2}\right]_{(\mathrm{PDMS})} /\left[\mathrm{Br}_{2}\right]_{(\mathrm{aq})}$ of approximately 400 . The large value of the partition coefficient implies that bromine readily enters the PDMS from the BZ solution and, hence, supports our hypothesis of a geometry-controlled activation via loss of bromine.

Although the partition coefficient yields useful information on the possible extent of the bromine loss, it does not provide insight into the dynamical aspects of this process. The literature indicates that compounds such as cyclohexane diffuse rapidly through PDMS. Lu et al. reported diffusion constants of $1.4 \times$ $10^{-5}$ to $2.2 \times 10^{-5} \mathrm{~cm}^{2} / \mathrm{s}$ for oxygen diffusion in silica-filled and unfilled PDMS films at room temperature. ${ }^{33}$ Buraphacheep et al. measured slightly smaller diffusion constants for various alcohols. ${ }^{34}$ For the case of PDMS elastomers created from the Sylgard 184 kit, it is important to note that the starting material may contain oligomers with terminal $\mathrm{Si}-\mathrm{H}$ and $\mathrm{Si}-\mathrm{C}=\mathrm{C}$ groups that can possibly react with bromine in the illuminated samples. Consequently, we expect passive transport of bromine through our PDMS matrices that is potentially complicated by reaction kinetics.

Chemical Waves in Complex Arrays. Soft lithography provides the ability to manufacture complex reaction arrays very easily. In the framework of our methodology, these arrays can consists of structures smaller than $50 \mu \mathrm{m}$. An example of a $2 \mathrm{D}$ array is shown in Figure 6. The profilometric data show a small section $\left(4 \mathrm{~mm}^{2}\right)$ of a PDMS microdevice consisting of periodically spaced obstacles. These obstacles have a height of $80 \mu \mathrm{m}$ and a side length of $500 \mu \mathrm{m}$. After chemically sealing the microdevice with a glass lid, it is loaded with BZ solution. The resulting reactor creates a heterogeneous excitable medium that consists of a network of thin conduits. The cross section of these channels is slightly trapezoidal with a width of $200 \mu \mathrm{m}$ at halfheight.

Figure 7 illustrates the evolution of a solitary excitation pulse within the microdevice. The complex $\mathrm{Fe}(\mathrm{II})\left[\mathrm{batho}\left(\mathrm{SO}_{3}\right)_{2}\right]_{3}$ is employed as the catalyst. Figure 7 , frames $\mathrm{A}-\mathrm{E}$ show a sequence of consecutive snapshots in which the darkest regions correspond to the reduced BZ system. The gray levels in frames $\mathrm{F}-\mathrm{J}$ are extracted from the same data but correspond to the local change in absorption with bright areas, indicating rapid oxidation of the catalyst. The sequence shows a traveling pulse that nucleated spontaneously at the intersection of two channels. Although this front would follow an ideal circle in a spatially homogeneous reaction system, it is strongly deformed in our microdevice. The expanding front consists of an increasing number of individual segments that create an overall diamondshaped geometry. This segmentation and deformation of the excitation pulse is caused by the specific geometry of the microdevice that grants unobstructed propagation in the vertical and horizontal directions only.

The geometry of the reactor grid also affects the dynamics of rotating spiral waves. The image frames presented in Figure 8 show a single clockwise-rotating spiral wave in a PDMS-BZ device identical to the one in Figures 6 and 7. In spatially homogeneous systems, the shape of these vortices is well described by an Archimedean spiral. ${ }^{35}$ Here, however, we find

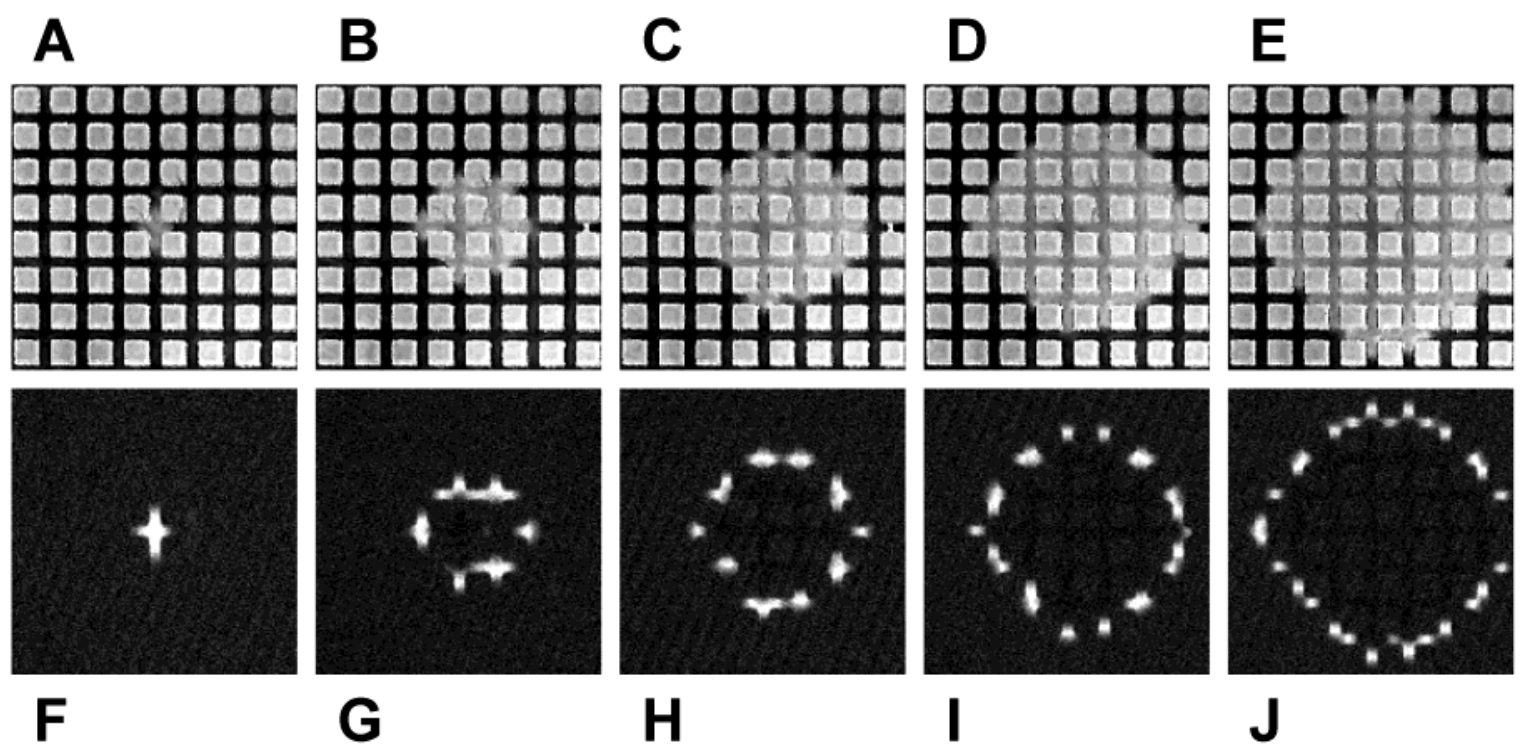

Figure 7. Target pattern in a grid-style microreactor. The images $(\mathrm{A}-\mathrm{E})$ are snapshots taken every $20 \mathrm{~s}$. The difference images $(\mathrm{F}-\mathrm{J})$ are taken every $20 \mathrm{~s}$ and are computed as $G(x, y, t)-G(x, y, t-\Delta t)$ with $\Delta t=10 \mathrm{~s}$ and $G$ denoting the gray level of a pixel site $(x, y)$ at a given time $t$. Accordingly, the bright areas in $(\mathrm{F}-\mathrm{J})$ indicate the front of the traveling oxidation wave. Initial $\mathrm{BZ}$ concentrations are $0.25 \mathrm{M} \mathrm{H}_{2} \mathrm{SO}_{4}, 0.15 \mathrm{M} \mathrm{CHD}$ $0.10 \mathrm{M} \mathrm{NaBrO}_{3}$, and $3.1 \mathrm{mM} \mathrm{Fe}(\mathrm{II})\left[\text { batho( }\left(\mathrm{SO}_{3}\right)_{2}\right]_{3}$. The field of view is $6 \times 6 \mathrm{~mm}^{2}$. 


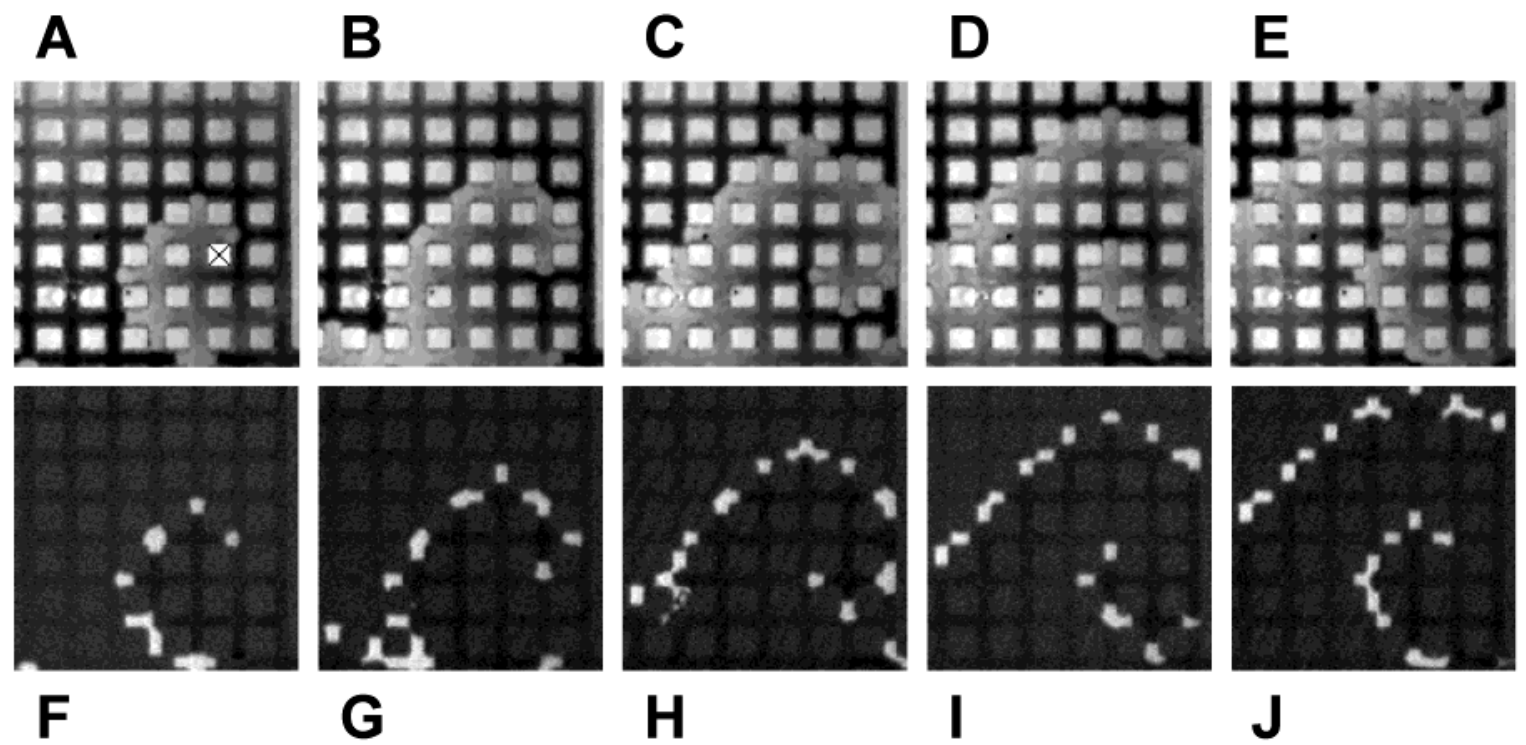

Figure 8. Motion of a single, clockwise-rotating spiral wave in a grid-style microreactor. The spatial images (A-E) are snapshots taken every 20 s. The spiral tip travels around a single PDMS obstacle, marked with an " $\times$ " in image (A). The image data (F-J) are calculated from $(\mathrm{A}-\mathrm{E})$ by image subtraction $(\Delta t=10 \mathrm{~s})$. Initial $\mathrm{BZ}$ concentrations are $0.50 \mathrm{M} \mathrm{H}_{2} \mathrm{SO}_{4}, 0.15 \mathrm{M} \mathrm{CHD}, 0.10 \mathrm{M} \mathrm{NaBrO}_{3}$, and $3.1 \mathrm{mM} \mathrm{Fe}(\mathrm{II})\left[\mathrm{batho}(\mathrm{SO})_{2}\right]_{3}$. The image area is $5 \times 5 \mathrm{~mm}^{2}$.

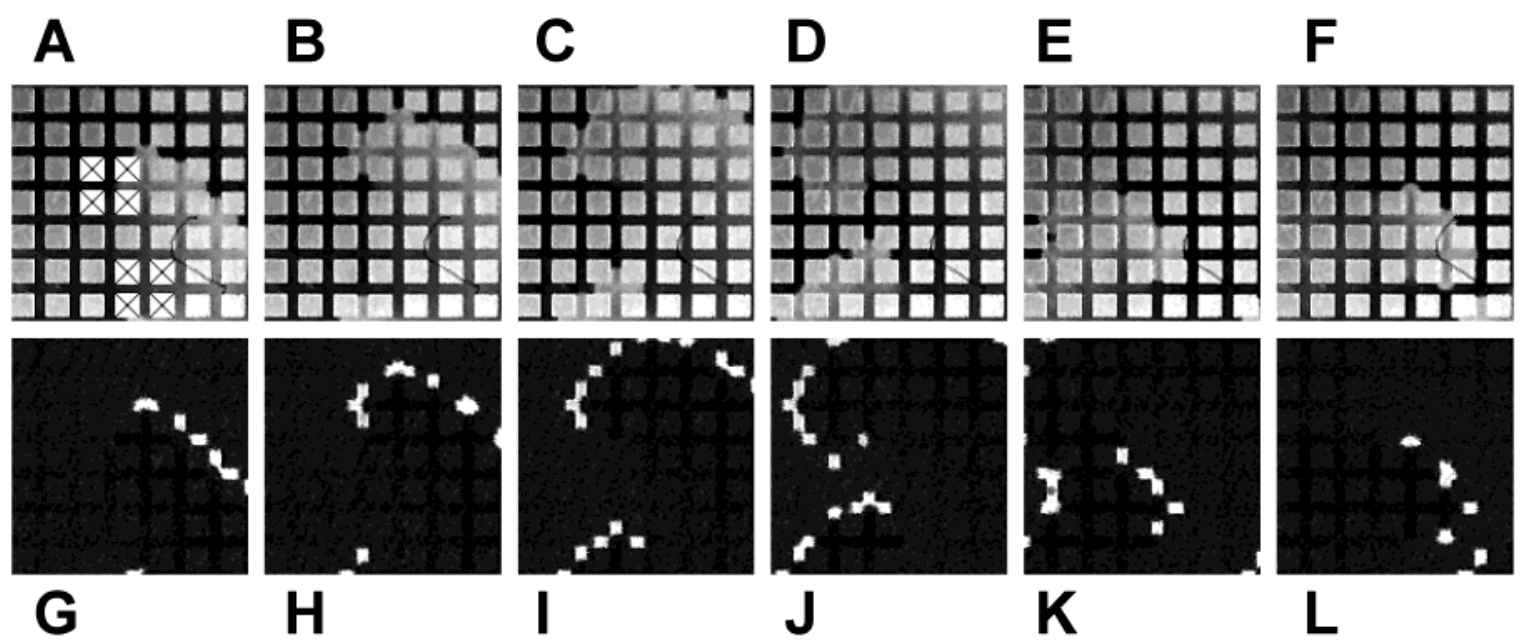

Figure 9. Evolution of a pair of rotating spiral waves in a grid-style microreactor. The images (A-F) are snapshots taken every $30 \mathrm{~s}$. The spiral tips travel around a group of four PDMS obstacles, marked with an " $\times$ " on each of the four squares in image (A). The difference images $(\mathrm{G}-\mathrm{L})$ are taken every $30 \mathrm{~s}$ with the frame taken $10 \mathrm{~s}$ prior subtracted. Initial $\mathrm{BZ}$ concentrations are $0.50 \mathrm{M} \mathrm{H}_{2} \mathrm{SO}_{4}, 0.15 \mathrm{M} \mathrm{CHD}, 0.10 \mathrm{M} \mathrm{NaBrO}$, and $3.1 \mathrm{mM} \mathrm{Fe}(\mathrm{II})\left[\text { batho }\left(\mathrm{SO}_{3}\right)_{2}\right]_{3}$. The image area is $5 \times 5 \mathrm{~mm}^{2}$.

a pronounced elongation in the vertical and horizontal directions that is analogous to the one described above. Moreover, the spiral in Figure 8 is rotating around one particular PDMS obstacle. A similar pinning of spiral waves at an artificial, unexcitable core has been observed in earlier experiments with spiral waves in a photosensitive BZ system ${ }^{19}$ and, hence, is not unexpected. However, we will describe in the following the fact that this phenomenon can also involve multiobstacle clusters.

Because the reaction system does not provide a steady inflow of reactants, the concentrations of bromate and 1,4-cyclohexanedione slowly decrease over the course of time. This depletion of substrates induces slow transients in the wave dynamics that typically lead to an increase in the wavelength (i.e., pitch) and the period of spiral waves. Moreover, the tip trajectory of the aging spiral is expected to expand into larger areas. Figure 9 illustrates the dynamics of spiral waves at a later stage of the experiment shown in Figure 8. Figure 9 reveals a pair of counterrotating spirals that indeed have an increased wavelength. At this point, it is important to note that earlier waves traversed the entire solution-loaded area of the microdevice. Hence, the dynamics are not compromised by imperfections in the reactor matrix. Moreover, no changes due to precipitation or gas bubble formation are detected. Nonetheless, the tips of both spirals in Figure 9 are pinned to clusters of $2 \times 2$ obstacles. The global wave period in this experiment corresponds to the completion of a full rotation of the tip around the perimeter of a $2 \times 2$ cluster. As the spiral tip moves along this perimeter, it encounters periodically the channels separating the four obstacles and penetrates them to a certain distance. For both spirals, however, this penetration fails at the central intersection of the cluster.

Figure 10 reveals that this unusual type of spiral rotation gives rise to two self-organized cores within the microdevice. For each position, the plot depicts the maximum gray level detected during the course of two rotation periods $(300 \mathrm{~s})$. The PDMS obstacles are the brightest areas whereas the BZ phase is slightly darker. At the center of the $2 \times 2$ clusters (cf. Figure 9), the maximum drops to low gray levels, indicating the absence of 


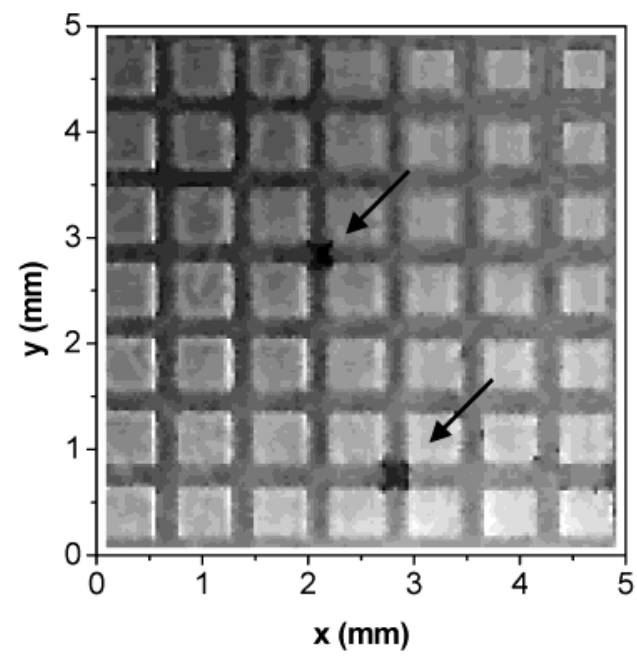

Figure 10. Analysis of the data shown in Figure 9 revealing two spiral cores (see arrows) at the intersections of BZ tracks. For each pixel, the image data represents the maximum gray level detected during two full rotational periods $(300 \mathrm{~s})$ of the spirals. The brightest regions correspond to the PDMS obstacles, whereas the spiral cores are small dark areas that do not undergo oxidation at any given time. The $\mathrm{x}$-shaped appearance of the cores is not an artifact but stems from repeated propagation failures of secondary spiral tips at the central intersections.

any oxidized phase. On the contrary, this type of analysis yields no dark areas in the case of spirals pinned to one obstacle only. In other words, the two dark areas in Figure 10 remain unexcited at all times and hence form an "eye-of-the-hurricane-like" spiral core.

\section{Discussion and Conclusions}

In summary, we have utilized soft lithography to create microfluidic devices for use with the $\mathrm{BZ}$ reaction. The substitution of the classic organic substrate malonic acid by 1,4cyclohexanedione is one of the essential steps in this lithographyassisted approach because it prevents the formation of undesired bubbles from gaseous reaction products. Our devices are shown to have an activating effect on chemical waves as the result of bromine diffusion into the PDMS. This transport is driven by the high solubility of bromine in PDMS. Consequently, this phenomenon could be used in future studies to create specific activation patterns that provoke spatial perturbations on exceedingly short length scales. It is also tempting to think of a possible bromine-mediated coupling between PDMS-separated BZ compartments. However, such attempts are likely to be hampered by the three-dimensionality of the elastomer matrix, which is expected to decrease the coupling strength by dilution. Nonetheless, it is worth mentioning that our basic methodology can be readily adjusted to other polymeric materials, which potentially opens avenues for future investigations that might allow the tailoring of specific types of coupling through nonaqueous phases.

It is furthermore important to note that in the case of PDMSBZ hybrids the loss of bromine from the aqueous BZ solution alters the overall dynamics of the reaction system, which creates the following undesired but benign problem. Chemical waves that propagate in our microdevices do not show the same features as waves in simple batch reactors manufactured from glass or plexiglass. Regardless of the channel pattern in a PDMS-BZ mirodevice, chemical waves are inherently close to the elastomer. Consequently, quantitative data on wave speeds and spiral wave parameters cannot be transferred between glass and PDMS reactors, although concentration-dependent trends are likely to persist.

In the framework of our investigation, the replacement of the classic BZ catalyst $\mathrm{Fe}(\mathrm{II})(\text { phen })_{3}$ (ferroin) by $\mathrm{Fe}$ (II)[batho$\left.\left(\mathrm{SO}_{3}\right)_{2}\right]_{3}$ proved to be a useful substitution. We expect that the resulting enhancement of absorption differences between the reduced and oxidized catalyst will aid future investigations significantly, in particular, those that involve increasingly smaller structures. Moreover, the $\mathrm{Fe}(\mathrm{II})\left[\mathrm{batho}\left(\mathrm{SO}_{3}\right)_{2}\right]_{3}$-catalyzed $\mathrm{BZ}$ reaction is a remarkably understudied system despite the fact that this complex is the only known negatively charged BZ catalyst. Because one can envision a methodological fusion of our approach and microchip-based electrophoresis devices, ${ }^{25}$ this feature might provide additional flexibility for experimental developments.

Last, we have demonstrated the effect of grid-type microdevices on target patterns and rotating spiral waves. The most striking phenomenon is the pronounced segmentation and deformation of these well-known excitation patterns. Similar observations have been reported by Suzuki et al. ${ }^{22}$ and Steinbock et al., ${ }^{18}$ who employed two alternate methodologies. Additionally, spiral waves tend to be pinned to individual units of the PDMS-based obstacles with a perimeter-dependent increase of the global rotation period. An intriguing finding of our study is the pinning of spiral waves to composite structures (i.e., structures that are not topologically connected; see Figures 9 and 10). Although unmentioned in the literature, this phenomenon can be anticipated if the tip trajectory of an unperturbed rigid-rotation spiral is larger than the perimeter of the PDMS obstacle. In our experiments, however, the spiral tip can perform sharp turns at the edges of the individual square obstacles and fails only at the central intersection of the $2 \times 2$ obstacle cluster. This seeming contradiction can be resolved by assuming that the spiral has undergone a Hopf bifurcation to a meandering motion $^{35-37}$ that involves rapid turns or wide arches in the spatially homogeneous system. Moreover, the observed propagation failure might be assisted by a reduced bromine loss at the cluster's central junction.

To resolve these intriguing questions, further systematic studies are necessary that go beyond the primary scope of this article. Our methodologies for the fabrication of PDMS-BZ microdevices, however, provide a powerful experimental tool for future investigations that should target a variety of intriguing problems concerning chemical and biological pattern formation in heterogeneous reaction-diffusion systems. ${ }^{38-40}$ In this respect, our study also closes the length-scale gap between the classic approach to exploring chemical self-organization in homogeneous media and recent efforts ${ }^{17}$ that target selforganization in heterogeneous micellar reaction systems. In conclusion, we believe that the current advances in microfluidics have the potential to contribute significantly to the field of nonlinear dynamics in chemistry and biology.

Acknowledgment. This work was supported by the National Science Foundation (NSF grant CHE-20023105). We thank Kenneth Weston for discussions.

\section{References and Notes}

(1) Chemical Waves and Patterns; Kapral, R.; Showalter, K., Eds.; Kluwer: Dordrecht, The Netherlands, 1995.

(2) Winfree, A. T. The Geometry of Biological Time; Springer: New York, 1980

(3) Zaikin, A. N.; Zhabotinsky, A. M. Nature 1970, 225, 535.

(4) Winfree, A. T. Science 1972, 175, 634.

(5) Kim, M.; Bertram, M.; Pollmann, M.; von Oertzen, A.; Mikhailov, A. S.; Rotermund, H. H.; Ertl, G. Science 2001, 292, 1357. 

8649.

(6) Field, R. J.; Noyes, R. M.; Körös, E. J. Am. Chem. Soc. 1972, 94,

(7) Marchant, J. S.; Ramos, V.; Parker, I. Am. J. Physiol.: Cell Physiol. 2002, 282, C1374

(8) Levine, H.; Reynolds, W. Phys. Rev. Lett. 1991, 66, 2400. 37.

(9) Müller, S. C.; Mair, T.; Steinbock, O. Biophys. Chem. 1998, 72,

(10) Steinbock, O.; Tóth, Á.; Showalter, K. Science 1995, 267, 868.

(11) Steinbock, O.; Kettunen, P.; Showalter, K. J. Phys. Chem. 1996, $100,18970$.

(12) Ichino, T.; Igarashi, Y.; Motoike, I. N.; Yoshikawa, K. J. Chem. Phys. 2003, 118, 8185 .

(13) Wolff, J.; Papathanasiou, A. G.; Kevrekidis, I. G.; Rotermund, H. H.; Ertl, G. Science 2001, 294, 134.

(14) Pollmann, M.; Rotermund, H. H.; Ertl, G.; Li, X. J.; Kevrekidis, I

G. Phys. Rev. Lett. 2001, 86, 6038 .

(15) Shvartsman, S. Y.; Schutz, E.; Imbihl, R.; Kevrekidis, I. G. Catal. Today 2001, 70, 301

(16) Toth, A.; Gaspar, V.; Showalter, K. J. Phys. Chem. 1994, 98, 522.

(17) Vanag, V. K.; Epstein, I. R. Science 2001, 294, 835.

(18) Steinbock, O.; Kettunen, P.; Showalter, K. Science 1995, 269, 1857.

(19) Steinbock, O.; Müller, S. C. Physica A 1992, 188, 61.

(20) Steinbock, O.; Zykov, V. S.; Müller, S. C. Phys. Rev. E 1993, 48 , 3295 .

(21) Sakurai, T.; Mihaliuk, E.; Chirila, F.; Showalter, K. Science 2002. 296, 2009

(22) Suzuki, K.; Yoshinobu, T.; Iwasaki, H. Jpn. J. Appl. Phys., Part 2 1999, 38, L345.

(23) Suzuki, K.; Yoshinobu, T.; Iwasaki, H. J. Phys. Chem. A 2000, 104,5154
(24) Suzuki, K.; Yoshinobu, T.; Iwasaki, H. J. Phys. Chem. A 2000, 104,6602 .

(25) McDonald, J. C.; Duffy, D. C.; Anderson, J. R.; Chiu, D. T.; Wu, H.; Schueller, O. J. A.; Whitesides, G. M. Electrophoresis 2000, 21, 27. (26) Deng.; T.; Wu, H.; Brittain, S. T.; Whitesides, G. M. Anal. Chem. 2000, 72,3176

(27) Kurin-Csörgei, K.; Zhabotinsky, A. M.; Orbán, M.; Epstein, I. R. J. Phys. Chem. 1996, 100, 5393.

(28) Manz, N.; Müller, S. C.; Steinbock, O. J. Phys. Chem. A 2000 104,5895 .

(29) Hamik, C. T.; Manz, N.; Steinbock, O. J. Phys. Chem. A 2001, 105,6144

(30) Yamaguchi, T.; Kuhnert, L.; Nagy-Ungvarai, Z.; Müller, S. C.; Hess, B. J. Phys. Chem. 1991, 95, 5831.

(31) Schilt, A. A. Analytical Application of 1,10-Phenanthroline and Related Compounds; Pergamon Press: Oxford, U.K., 1969.

(32) Steinbock, O.; Müller, S. C. J. Phys. Chem. A 1998, 102, 6485

(33) Lu, X.; Manners, I.; Winnik, M. A. Macromolecules 2001, 34, 1917.

(34) Buraphacheep, V.; Wurster, D. E.; Wurster, D. E. Pharm. Res. 1994 11,561

(35) Müller, S. C.; Plesser, T.; Hess, B. Physica D 1987, $24,87$.

(36) Steinbock, O.; Müller, S. C. Phys. Rev. E 1993, 47, 1506.

(37) Manz, N.; Ginn, B. T.; Steinbock, O. J. Phys. Chem. A 2003, 107, 11008.

(38) Xin, J. SIAM Rev. 2000, 42, 161.

(39) Yang, J. B.; Kalliadasis, S.; Merkin, J. H.; Scott, S. K. Chaos 2001, 11,479 .

(40) Bär, M.; Meron, E.; Utzny, C. Chaos 2002, 12, 204. 